\title{
Nonpoint Symmetry and Reduction of Nonlinear Evolution and Wave Type Equations
}

\author{
Ivan Tsyfra ${ }^{1}$ and Tomasz Czyżycki ${ }^{2}$ \\ ${ }^{1}$ AGH University of Science and Technology, Faculty of Applied Mathematics, 30 Mickiewicza Avenue, 30-059 Krakow, Poland \\ ${ }^{2}$ Institute of Mathematics, University of Białystok, Ciołkowskiego 1M, 15-245 Białystok, Poland \\ Correspondence should be addressed to Ivan Tsyfra; tsyfra@agh.edu.pl
}

Received 20 February 2015; Accepted 25 May 2015

Academic Editor: R. Naz

Copyright ( 2015 I. Tsyfra and T. Czyżycki. This is an open access article distributed under the Creative Commons Attribution License, which permits unrestricted use, distribution, and reproduction in any medium, provided the original work is properly cited.

\begin{abstract}
We study the symmetry reduction of nonlinear partial differential equations with two independent variables. We propose new ansätze reducing nonlinear evolution equations to system of ordinary differential equations. The ansätze are constructed by using operators of nonpoint classical and conditional symmetry. Then we find solution to nonlinear heat equation which cannot be obtained in the framework of the classical Lie approach. By using operators of Lie-Bäcklund symmetries we construct the solutions of nonlinear hyperbolic equations depending on arbitrary smooth function of one variable too.
\end{abstract}

\section{Introduction}

It is well known that the classical Lie symmetry method of point transformations is often used for reducing the number of independent variables in partial differential equation to obtain ordinary differential equations. After integration of reduced differential equations one can obtain partial solutions of the equation under study [1-3]. The main problem is that the maximal invariance group of point transformations of differential equations used in applications is not sufficiently wide and thus the group approach can not be successfully applied to these equations. The concept of generalized conditional symmetry has been introduced in $[4,5]$ to extend the applicability of the symmetry method to the construction of solutions of evolution equations. The relationship of generalized conditional symmetries of evolution equations to compatibility of system of differential equations is studied in [6]. The method for construction of nonlocally related partial differential equation systems for a given partial differential equation has been proposed in [7]. The starting point for the method is the existence of operator of point symmetry of the equation under study. Through nonlocally related systems one can construct operators of nonlocal symmetry and nonlocal conservation laws of initial equation.
We use operators of nonpoint classical and conditional symmetries to extend the class of differential equations to which the symmetry method is applicable. In this paper we study the symmetry reduction of partial differential equations with two independent variables by using the operators of nonpoint symmetry because the prolongated operators of classical point symmetry lead to the classical invariant solutions. The method can be naturally generalized to the multidimensional case. We construct the ansatz for dependent variable $u$ or its derivatives which reduces the scalar partial differential equation to a system of ordinary differential equations. We use the operators of the classical point symmetry $[1,2]$ of the corresponding system which are not the prolongated operators of point symmetries admitted by the original equation to construct the ansatz for derivatives. We construct the ansatz for $u$ by using ordinary differential equation admitting the operators of Lie-Bäcklund symmetry (in the classical sense $[2,4]$ ). We consider nonlinear evolution and wave type equations and present the operator of conditional symmetry for the corresponding system which generates the Bäcklund transformations for nonlinear wave equation.

Recall, that the well-known integrable nonlinear differential equations such as Korteweg-de-Vries, sine-Gordon, 
and cubic Schroedinger equations admit an infinite number of Lie-Bäcklund symmetry operators $[1,2]$. Another goal of this paper is to show that such important properties of nonlinear partial differential equations as existence of Bäcklund transformations, linearization, and existence of the class of solutions depending on arbitrary function can be related to their invariance under the finite number of nonpoint symmetry operators.

\section{Nonpoint Symmetry and Reduction of Nonlinear Wave Type and Evolution Equations with Two Independent Variables}

The concept of differential invariant solutions based on infinite Lie group $G$ is introduced in [3]. This group is a classical symmetry group of point transformations of dependent and independent variables for the equation under study. Generally speaking, analysis similar to that in constructing differential invariant solutions enables us to obtain the ansätze for derivatives $u_{x_{1}}, u_{x_{2}}$ by virtue of operators of nonpoint symmetry $[3,8]$. Let us consider nonlinear differential equation

$$
u_{x_{2} x_{2}}=\frac{1}{1-u_{x_{1}}^{r}}, \quad r \neq 0, \pm 1
$$

We search for the ansatz for the derivatives of such form

$$
\begin{aligned}
\frac{\partial u}{\partial x_{1}} & =R_{1}\left(x_{1}, x_{2}, u, \varphi_{1}(\omega), \varphi_{2}(\omega)\right), \\
\frac{\partial u}{\partial x_{2}} & =R_{2}\left(x_{1}, x_{2}, u, \varphi_{1}(\omega), \varphi_{2}(\omega)\right),
\end{aligned}
$$

where $\omega=\omega\left(x_{1}, x_{2}, u\right)$. Operators of classical and conditional symmetry of the corresponding system can be used to find $R_{1}$, $R_{2}$. The corresponding system has the form

$$
\begin{aligned}
& v_{2}^{1}=v_{1}^{2}, \\
& v_{2}^{2}=\frac{1}{1-\left(v^{1}\right)^{r}},
\end{aligned}
$$

where $v^{1}=u_{x_{1}}, v^{2}=u_{x_{2}}$, and $v_{k}^{i}=v_{x_{k}}^{i}, i, k=1,2$. To construct ansatz of type (2) we use the symmetry operator

$$
Q=(r+1) x_{1} \partial_{x_{1}}+r v^{2} \partial_{x_{2}}-v^{1} \partial_{v^{1}}+r v^{2} \partial_{v^{2}}
$$

of system (3). It is obvious that operator $Q$ generates nonpoint group transformations for variables $x_{1}, x_{2}, u$. It is easy to find the invariants of one-parameter Lie group with generator $Q$

$$
\begin{aligned}
\omega & =x_{2}-v^{2}, \\
\omega_{1} & =v^{1}\left(x_{1}\right)^{1 /(r+1)}, \\
\omega_{2} & =v^{2}\left(x_{1}\right)^{-r /(r+1)} .
\end{aligned}
$$

By using these invariants one can construct the ansatz for $v^{1}$, $v^{2}$ :

$$
\begin{aligned}
& v^{1}=\left(x_{1}\right)^{-1 /(r+1)} \varphi_{1}(\omega), \\
& v^{2}=\left(x_{1}\right)^{r /(r+1)} \varphi_{2}(\omega) .
\end{aligned}
$$

From (6) we have

$$
v_{2}^{2}=\frac{\left(x_{1}\right)^{r /(r+1)} \varphi_{2}^{\prime}}{1+\left(x_{1}\right)^{r /(r+1)} \varphi_{2}^{\prime}},
$$

where $\varphi_{2}^{\prime}=d \varphi_{2} / d \omega$. Substituting (6) and (7) into the equation

$$
v_{2}^{2}=\frac{1}{1-\left(v^{1}\right)^{r}}
$$

yields

$$
\left(x_{1}\right)^{r /(r+1)} \varphi_{2}^{\prime}-\varphi_{2}^{\prime} \varphi_{1}^{r}=1+\left(x_{1}\right)^{r /(r+1)} \varphi_{2}^{\prime} .
$$

Thus we get the first reduced ordinary differential equation

$$
\varphi_{2}^{\prime} \varphi_{1}^{r}=-1
$$

The second one we obtain from the compatibility condition $v_{2}^{1}=v_{1}^{2}$. It has the form

$$
\frac{r}{r+1} \varphi_{2}=\varphi_{1}^{\prime}
$$

We take the particular solution of reduced system of ordinary differential equations (10) and (11) in the form

$$
\begin{aligned}
& \varphi_{1}=\left(\sqrt{\frac{r(r+1)}{2(r-1)}} \omega+C_{1}\right)^{2 /(r+1)}, \\
& \varphi_{2}=\sqrt{\frac{2(r+1)}{r(r-1)}}\left(\sqrt{\frac{r(r+1)}{2(r-1)}} \omega+C_{1}\right)^{(1-r) /(r+1)},
\end{aligned}
$$

where $C_{1}=$ const. Thus one has to integrate overdetermined compatible system of differential equations

$$
\begin{aligned}
& u_{x_{1}}=\left(x_{1}\right)^{-1 /(r+1)}\left(\sqrt{\frac{r(r+1)}{2(r-1)}}\left(x_{2}-u_{x_{2}}\right)\right. \\
& \left.+C_{1}\right)^{2 /(r+1)}, \\
& u_{x_{2}}=\left(x_{1}\right)^{r /(r+1)} \sqrt{\frac{2(r+1)}{r(r-1)}}\left(\sqrt{\frac{r(r+1)}{2(r-1)}}\left(x_{2}-u_{x_{2}}\right)\right. \\
& \left.+C_{1}\right)^{(1-r) /(r+1)}
\end{aligned}
$$


to construct the solution of (1). Nevertheless it is easy to get the solution of the equation

$$
w_{t}-\frac{1}{r}\left(\frac{(w-1)^{(1-r) / r} w_{x}}{w^{(1+r) / r}}\right)_{x}=0
$$

in such form

$$
\begin{aligned}
& \left(1-\frac{1}{w}\right)^{1 / r}=(t)^{-1 /(r+1)} \\
& \cdot\left(\sqrt{\frac{r(r+1)}{2(r-1)}}(x-\theta)+C_{1}\right)^{2 /(r+1)}, \\
& \theta=(t)^{r /(r+1)} \\
& \cdot \sqrt{\frac{2(r+1)}{r(r-1)}}\left(\sqrt{\frac{r(r+1)}{2(r-1)}}(x-\theta)+C_{1}\right)^{(1-r) /(r+1)}
\end{aligned}
$$

The tangent transformations groups are also used in the framework of this approach. Let us consider the nonlinear evolution equation

$$
u_{t}=e^{1 / u_{x x}} .
$$

One can construct operator of tangent transformations of the form

$$
K=-t \partial_{t}+u_{x} \partial_{x}+\frac{u_{x}^{2}}{2} \partial_{u}+u_{t} \partial_{u_{t}}
$$

admitted by (16). The first order functionally independent differential invariants of the corresponding one-parameter Lie group of tangent transformations can be chosen in the form

$$
\begin{aligned}
& \omega=x u_{x}-2 u, \\
& \omega_{1}=\ln u_{t}-\frac{x}{u_{x}}, \\
& \omega_{2}=u_{x}, \\
& \omega_{3}=t u_{t} .
\end{aligned}
$$

In order to construct ansatz of type (2) reducing (16) to system of ordinary differential equations we consider twodimensional Lie algebra with basic operators $\left\{K, P_{t}=\partial / \partial t\right\}$. The operators satisfy the commutation relation $\left[K, P_{t}\right]=P_{t}$. The invariants of two-parameter Lie group with generators $K, P_{t}$ are $\omega, \omega_{1}$, and $\omega_{2}$. Then we construct the ansatz by using these invariants in the form

$$
\begin{aligned}
& u_{x}=f(\omega), \\
& u_{t}=\exp \left(\varphi(\omega)+\frac{x}{f(\omega)}\right) .
\end{aligned}
$$

From (19) and (16) we have

$$
u_{x x}=-\frac{f^{\prime} f}{1-x f^{\prime}}
$$

and first ordinary differential equation

$$
f^{\prime} f \varphi=-1 \text {. }
$$

From the condition $u_{x t}=u_{t x}$ it follows that $f, \varphi$ satisfy the second ordinary differential equation

$$
\frac{f-\varphi^{\prime} f^{3}}{f^{2}}=-2 f^{\prime} .
$$

Thus the reduced system consists of (21) and (22). From (21), (22), and (19) it follows that the solutions of (16) can be constructed by integrating overdetermined compatible system

$$
\begin{aligned}
& u_{t}=\exp \left[\frac{2\left(\sqrt{C_{1}-4\left(x u_{x}-2 u\right)}+x\right)}{2 C_{2}-\sqrt{C_{1}-4\left(x u_{x}-2 u\right)}}\right], \\
& u_{x}=\frac{2 C_{2}-\sqrt{C_{1}-4\left(x u_{x}-2 u\right)}}{2},
\end{aligned}
$$

where $C_{1}, C_{2}$ are arbitrary real constants.

Next we emphasize that the operators of conditional symmetry of corresponding system can be used for construction the Bäcklund transformations for nonlinear wave equation

$$
u_{x_{1} x_{2}}=\left[1-k^{2} u_{x_{2}}^{2}\right]^{1 / 2} \sin u
$$

Indeed we showed that

$$
Q=\partial_{x_{3}}+k \cos x_{3} \partial_{v^{1}}+k^{-1} \sqrt{1-k^{2}\left(v^{2}\right)^{2}} \partial_{v^{2}}
$$

is the operator of conditional symmetry of the corresponding system

$$
\begin{aligned}
& v_{2}^{1}+v_{3}^{1} v^{2}=v_{1}^{2}+v_{3}^{2} v^{1}, \\
& v_{1}^{2}+v_{3}^{2} v^{1}=\sqrt{1-k^{2}\left(v_{2}\right)^{2}} \sin x_{3},
\end{aligned}
$$

where $u \equiv x_{3}$. Using operator $Q$ we can write the ansatz in the following form:

$$
\begin{aligned}
& u_{x_{1}}=\varphi_{2}+k \sin u, \\
& u_{x_{2}}=k^{-1} \sin \left(u-\varphi_{1}\right),
\end{aligned}
$$

where $\varphi_{1}, \varphi_{2}$ are unknown functions on $x_{1}, x_{2}$ and hence the Bäcklund transforms

$$
\begin{aligned}
& u_{x_{2}}=k^{-1} \sin (u-w), \\
& u_{x_{1}}=w_{x_{1}}+k \sin u
\end{aligned}
$$

relating (24) and sine-Gordon equation $w_{x_{1} x_{2}}=\sin w$. These Bäcklund transforms (28) have been obtained for the first time in [9] by another technique. 
Note that this approach is also applicable for linearization of nonlinear partial differential equations with two independent variables. Indeed, consider the second-order differential equation

$$
u_{x_{0} x_{0}}=F\left(u_{x_{0} x_{1}}, u_{x_{1} x_{1}}\right),
$$

where $F$ is a smooth function. Using the invariance of (29) under Lie group of transformations with corresponding fivedimensional Lie algebra given by basic elements $\partial_{x_{0}}, \partial_{x_{1}} \partial_{u}$, $x_{0} \partial_{u}$, and $x_{1} \partial_{u}$ we write the corresponding system in the form

$$
\begin{aligned}
\frac{\partial F}{\partial v^{2}} \frac{\partial v^{2}}{\partial x_{1}}+\frac{\partial F}{\partial v^{3}} \frac{\partial v^{3}}{\partial x_{1}} & =\frac{\partial v^{2}}{\partial x_{0}}, \\
\frac{\partial v^{3}}{\partial x_{0}} & =\frac{\partial v^{2}}{\partial x_{1}}, \\
v^{1} & =F\left(v^{2}, v^{3}\right),
\end{aligned}
$$

where $u_{x_{0} x_{0}} \equiv v^{1}\left(x_{0}, x_{1}\right), u_{x_{0} x_{1}} \equiv v^{2}\left(x_{0}, x_{1}\right)$, and $u_{x_{1} x_{1}} \equiv$ $v^{3}\left(x_{0}, x_{1}\right)$. One can prove that (30) possesses infinite Lie classical symmetry and can be linearized by hodograph transformations. Thus we obtained the method of linearization of the second-order partial differential equation of the form (29) for arbitrary function $F$.

Let us note that the symmetry group of corresponding system written in the general form contains the symmetry group of point transformations of initial equation as a subgroup and generators of point transformations can be used to construct ansatz (2). However these operators lead to invariant solutions in the classical Lie sense. We shall illustrate this property by the following example. Let us consider the wave equation

$$
u_{x_{1} x_{2}}=F(u),
$$

where $F$ is a smooth function. It is invariant with respect to the three-parameter Lie group. The basis of Lie algebra is given by $\left\{\partial_{x_{1}}, \partial_{x_{2}}, x_{1} \partial_{x_{1}}-x_{2} \partial_{x_{2}}\right\}$. Consider two-dimensional subalgebra with basic elements $\left\{\partial_{x_{2}}, x_{1} \partial_{x_{1}}-x_{2} \partial_{x_{2}}\right\}$. By using the differential invariants $u, x_{1} u_{x_{1}}$, and $u_{x_{2}} / x_{1}$ of the corresponding two-parameter Lie group we construct ansatz of the form

$$
\begin{aligned}
& u_{x_{1}}=\frac{f(u)}{x_{1}}, \\
& u_{x_{2}}=x_{1} \varphi(u)
\end{aligned}
$$

which reduces (31) to the system

$$
f^{\prime} \varphi=\varphi+\varphi^{\prime} f=F(u) .
$$

Let $F(u)=0$. Then we obtain two cases

$$
\begin{aligned}
\text { (1) } f^{\prime} & =0, \\
\varphi+\varphi^{\prime} f & =0
\end{aligned}
$$

and solution of reduced system has the form

$$
\begin{aligned}
f & =C_{1}=\text { const, } \\
\varphi & =C_{2} \exp \left(-\frac{u}{C_{1}}\right), \\
C_{2} & =\text { const. }
\end{aligned}
$$

By integrating system

$$
\begin{aligned}
\frac{\partial u}{\partial x_{1}} & =\frac{C_{1}}{x_{1}}, \\
\frac{\partial u}{\partial x_{2}} & =C_{2} x_{1} \exp \left(-\frac{u}{C_{1}}\right)
\end{aligned}
$$

one obtains the solution

$$
u=C_{1} \ln \left(\frac{C_{2}}{C_{1}} x_{1} x_{2}+C_{3} x_{1}\right),
$$

where $C_{3}$ is arbitrary real constant and $C_{1} \neq 0$, of (31) with $F=0$. In the second case we have

$$
\text { (2) } \begin{aligned}
\varphi & =0, \\
u_{x_{1}} & =\frac{1}{x_{1}} f(u), \\
u_{x_{2}} & =0
\end{aligned}
$$

and solution has the form

$$
u=h\left(x_{1}\right),
$$

where $h\left(x_{1}\right)$ is arbitrary differentiable function. Let us consider the operator

$$
Q=\alpha \partial_{x_{2}}+\beta\left(x_{1} \partial_{x_{1}}-x_{2} \partial_{x_{2}}\right),
$$

where $\alpha, \beta$ are arbitrary real constants. One can verify that

$$
Q\left(u-C_{1} \ln \left(\frac{C_{2}}{C_{1}} x_{1} x_{2}+C_{3} x_{1}\right)\right)=0
$$

if and only if

$$
\alpha \frac{C_{2}}{C_{1}}+\beta C_{3}=0
$$

It means that solution (37) is invariant with respect to one-dimensional subgroup of symmetry group of (31) with generator $Q$ where $\alpha, \beta$ satisfy condition (42). It is obvious that the solution (39) is invariant with respect to oneparameter group with generator $Q=\alpha \partial_{x_{2}}(\beta=0)$. Thus we conclude that any solution of (31) when $F=0$ constructed by this method with the help of two-dimensional Lie algebra with basic elements $\left\{\partial_{x_{2}}, x_{1} \partial_{x_{1}}-x_{2} \partial_{x_{2}}\right\}$ is an invariant one in the classical Lie sense.

Further we show how the operators of Lie-Bäcklund symmetry [2, 4] are used for reducing partial differential equations. Let us consider equation

$$
U(x, u, \underset{1}{u,}, \underset{2}{,}, \ldots, \underset{k}{u})=0
$$


where $x=\left(x_{1}, x_{2}, \ldots, x_{n}\right), u=u(x) \in C^{k}\left(\mathbb{R}^{n}, \mathbb{R}^{1}\right)$, and $u$ denotes all partial derivatives of $k$ th order and the $m$ th order ordinary differential equation of the form

$$
H\left(x_{1}, x_{2}, \ldots, x_{n}, u, \frac{\partial u}{\partial x_{1}}, \ldots, \frac{\partial^{m} u}{\partial x_{1}^{m}}\right)=0 .
$$

Let

$$
u=F\left(x, C_{1}, \ldots, C_{m}\right)
$$

where $F$ is a smooth function on variables $x, C_{1}, \ldots, C_{m}$ and $C_{1}, \ldots, C_{m}$ are arbitrary functions on variables $x_{2}, x_{3}, \ldots, x_{n}$, be a general solution of (44).

We use Theorem 1 from [10] which implies that if (44) is invariant with respect to the Lie-Bäcklund operator $X=$ $U(x, u, \underset{1}{u}, \underset{2}{u}, \ldots, \underset{k}{u}) \partial_{u}$ then the ansatz

$$
u=F\left(x, \varphi_{1}, \varphi_{2}, \ldots, \varphi_{m}\right)
$$

where $\varphi_{1}, \varphi_{2}, \ldots, \varphi_{m}$ depend on $n-1$ variables $x_{2}, x_{3}, \ldots, x_{n}$, reduces partial differential equation (43) to the system of $k_{1}$ equations for unknown functions $\varphi_{1}, \varphi_{2}, \ldots, \varphi_{m}$ with $n-1$ independent variables and $k_{1} \leq m$. We show the application of the theorem to nonlinear partial differential equation. Consider linear ordinary differential equation

$$
u_{x_{1} x_{1}}+\alpha^{2} u_{x_{1}}=0
$$

where $\alpha=$ const. Recall that the concepts of local theory of differential equations such as symmetry, conditional symmetry, conservation laws, and Lax representations are defined by differential equalities which must be satisfied only for solutions of the equations under study. One can prove that (47) admits the following Lie-Bäcklund operator:

$$
X=\left(u_{x_{1} x_{2}}-u_{x_{1}} F\left(u_{x_{1}}+\alpha^{2} u\right)\right) \partial_{u}
$$

where $F \in C^{2}\left(\mathbb{R}^{1}, \mathbb{R}^{1}\right)$. It means that the following criterium of invariance

$$
\begin{aligned}
X_{(2)}\left(u_{x_{1} x_{1}}+\alpha^{2} u_{x_{1}}\right)=0 & \\
& \text { whenever } u_{x_{1} x_{1}}+\alpha^{2} u_{x_{1}}=0,
\end{aligned}
$$

where $X_{(2)}$ is the prolongated operator of the second order [2], is fulfilled. We have proved that (47) admits operator of nonpoint (tangent) symmetry

$$
X_{1}=f\left(u, u_{x}\right) \partial u
$$

if $f\left(u, u_{x}\right)$ satisfies the following equation:

$$
f_{u u}^{\prime \prime}-2 \alpha^{2} f_{u u_{x}}^{\prime \prime}+\alpha^{4} f_{u_{x} u_{x}}^{\prime \prime}=0 .
$$

The general solution of this equation has the form

$$
f=A\left(u_{x_{1}}+\alpha^{2} u\right) u+B\left(u_{x_{1}}+\alpha^{2} u\right),
$$

where $A, B$ are arbitrary smooth functions of one variable. One can verify that (47) also admits operator

$$
X_{2}=e^{-\alpha^{2} x_{1}} h\left(u_{x_{1}}+\alpha^{2} u\right) \partial u
$$

where $h$ is arbitrary function on variable $u_{x_{1}}+\alpha^{2} u$. Then the ansatz

$$
u=\varphi_{1}\left(x_{2}\right)+e^{-\alpha^{2} x_{1}} \varphi_{2}\left(x_{2}\right)
$$

obtained from the general solution of (47) reduces wave type partial differential equations

$$
\begin{aligned}
u_{x_{1} x_{2}}= & u_{x_{1}} F\left(u_{x_{1}}+\alpha^{2} u\right)+A\left(u_{x_{1}}+\alpha^{2} u\right) u \\
& +B\left(u_{x_{1}}+\alpha^{2} u\right)+k u_{x_{2}} \\
& +e^{-\alpha^{2} x_{1}} h\left(u_{x_{1}}+\alpha^{2} u\right),
\end{aligned}
$$

where $k$ is a real constant. In general, the $x_{1}$ dependent coefficients in partial differential equations enable us to study the effects of field gradients.

Substituting (54) into (55) we obtain the reduced system of two ordinary differential equations

$$
\begin{aligned}
& -\alpha^{2} \varphi_{2}^{\prime} \\
& =-\alpha^{2} \varphi_{2} F\left(\alpha^{2} \varphi_{1}\right)+A\left(\alpha^{2} \varphi_{1}\right) \varphi_{2}+k \varphi_{2}^{\prime} \\
& +h\left(\alpha^{2} \varphi_{1}\right) \\
& A\left(\alpha^{2} \varphi_{1}\right) \varphi_{1}+B\left(\alpha^{2} \varphi_{1}\right)+k \varphi_{1}^{\prime}=0
\end{aligned}
$$

for unknown functions $\varphi_{1}\left(x_{2}\right), \varphi_{2}\left(x_{2}\right)$. One can obtain partial solutions of (55) from solutions of system (56). In particular, if $A=B=0$ and $k=0$ then system (56) is reduced to one ordinary differential equation of the form

$$
\varphi_{2}^{\prime}=\varphi_{2} F\left(\alpha^{2} \varphi_{1}\right)-\frac{1}{\alpha^{2}} h\left(\alpha^{2} \varphi_{1}\right)
$$

This equation is integrable by quadratures for arbitrary $\varphi_{1}\left(x_{2}\right)$. Its general solution has the form

$$
\begin{aligned}
\varphi_{2}= & \left(C_{1}-\frac{1}{\alpha^{2}} \int h\left(\alpha^{2} \varphi_{1}\left(x_{2}\right)\right) H\left(x_{2}\right) d x_{2}\right) \\
& \cdot \exp \left(\int F\left(\alpha^{2} \varphi_{1}\left(x_{2}\right)\right) d x_{2}\right),
\end{aligned}
$$

where $C_{1}=$ const,

$$
H\left(x_{2}\right)=\exp \left(-\int F\left(\alpha^{2} \varphi_{1}\left(x_{2}\right)\right) d x_{2}\right) .
$$

Using (54) one can construct the solution of nonlinear wave equation

$$
u_{x_{1} x_{2}}=u_{x_{1}} F\left(u_{x_{1}}+\alpha^{2} u\right)+e^{-\alpha^{2} x_{1}} h\left(u_{x_{1}}+\alpha^{2} u\right)
$$


in the following form:

$$
\begin{gathered}
u=\varphi_{1}\left(x_{2}\right)+\left(C_{1}-\frac{1}{\alpha^{2}} \int h\left(\alpha^{2} \varphi_{1}\left(x_{2}\right)\right) H\left(x_{2}\right) d x_{2}\right) \\
\cdot \exp \left(\int F\left(\alpha^{2} \varphi_{1}\left(x_{2}\right)\right) d x_{2}-\alpha^{2} x_{1}\right)
\end{gathered}
$$

where $\varphi_{1}\left(x_{2}\right)$ is arbitrary smooth function. So in the framework of this approach we have constructed solution with arbitrary function $\varphi_{1}\left(x_{2}\right)$ to nonlinear wave type partial differential equation (60) for arbitrary functions $F$ and $h$.

\section{Conclusions}

We have constructed ansätze (6) and ansätze (19) which reduce nonlinear evolution equations (1) and (16) to ordinary differential equations and can not be obtained by using classical Lie method. We have found the solution of nonlinear heat equation (14). It turns out that some of these ansätze result in the classical invariant solutions. Obviously, one can construct such ansätze by prolongated operators of point symmetry admitted by the initial equation but they lead to the invariant solutions too. It is necessary that operators of nonpoint and conditional symmetry should be applied to obtain new results.

As was noted above the linearization of class of nonlinear partial differential equations (29) is possible in the framework of this approach.

Finally we show that the existence of even at least one operator of Lie-Bäcklund symmetry to ordinary differential equations (47) gives the possibility of constructing solutions (61) defined by arbitrary functions to (60). To our knowledge the inverse scattering tranformation method is not applicable in this case.

\section{Conflict of Interests}

The authors declare that there is no conflict of interests regarding the publication of this paper.

\section{References}

[1] G. W. Bluman and S. Kumei, Symmetries and Differential Equations, vol. 81 of Applied Mathematical Sciences, Springer, New York, NY, USA, 1989.

[2] P. J. Olver, Applications of Lie Groups to Differential Equations, vol. 107 of Graduate Texts in Mathematics, Springer, New York, NY, USA, 1986.

[3] L. V. Ovsiannikov, Group Analysis of Differential Equations, Academic Press, New York, NY, USA, 1982.

[4] A. S. Fokas and Q. M. Liu, "Nonlinear interaction of traveling waves of nonintegrable equations," Physical Review Letters, vol. 72, no. 21, pp. 3293-3296, 1994.

[5] R. Z. Zhdanov, "Conditional Lie-Bäcklund symmetry and reduction of evolution equations," Journal of Physics. A. Mathematical and General, vol. 28, no. 13, pp. 3841-3850, 1995.

[6] M. Kunzinger and R. O. Popovych, "Generalized conditional symmetries of evolution equations," Journal of Mathematical Analysis and Applications, vol. 379, no. 1, pp. 444-460, 2011.
[7] G. W. Bluman and Z. Yang, "A symmetry-based method for constructing nonlocally related partial differential equation systems," Journal of Mathematical Physics, vol. 54, no. 9, Article ID 093504, 22 pages, 2013.

[8] I. Tsyfra, A. Napoli, A. Messina, and V. Tretynyk, "On new ways of group methods for reduction of evolution-type equations," Journal of Mathematical Analysis and Applications, vol. 307, no. 2, pp. 724-735, 2005.

[9] R. K. Dodd and R. K. Bullough, "Bäcklund transformations for the sine-Gordon equations," Proceedings of the Royal Society A: Mathematical, Physical and Engineering Sciences, vol. 351, no. 1667, pp. 499-523, 1976.

[10] I. M. Tsyfra, "Symmetry reduction of nonlinear differential equations," Proceedings of Institute of Mathematics, vol. 50, pp. 266-270, 2004. 


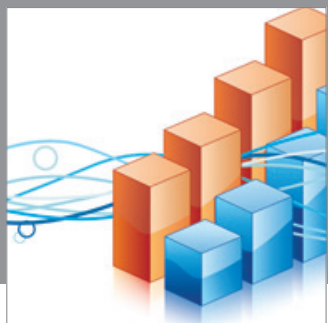

Advances in

Operations Research

mansans

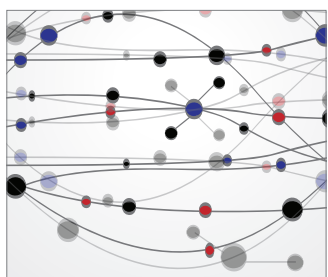

The Scientific World Journal
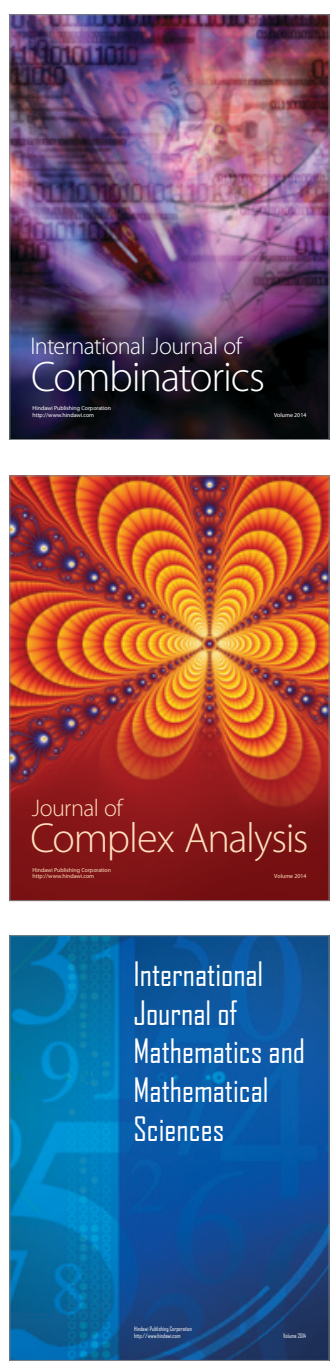
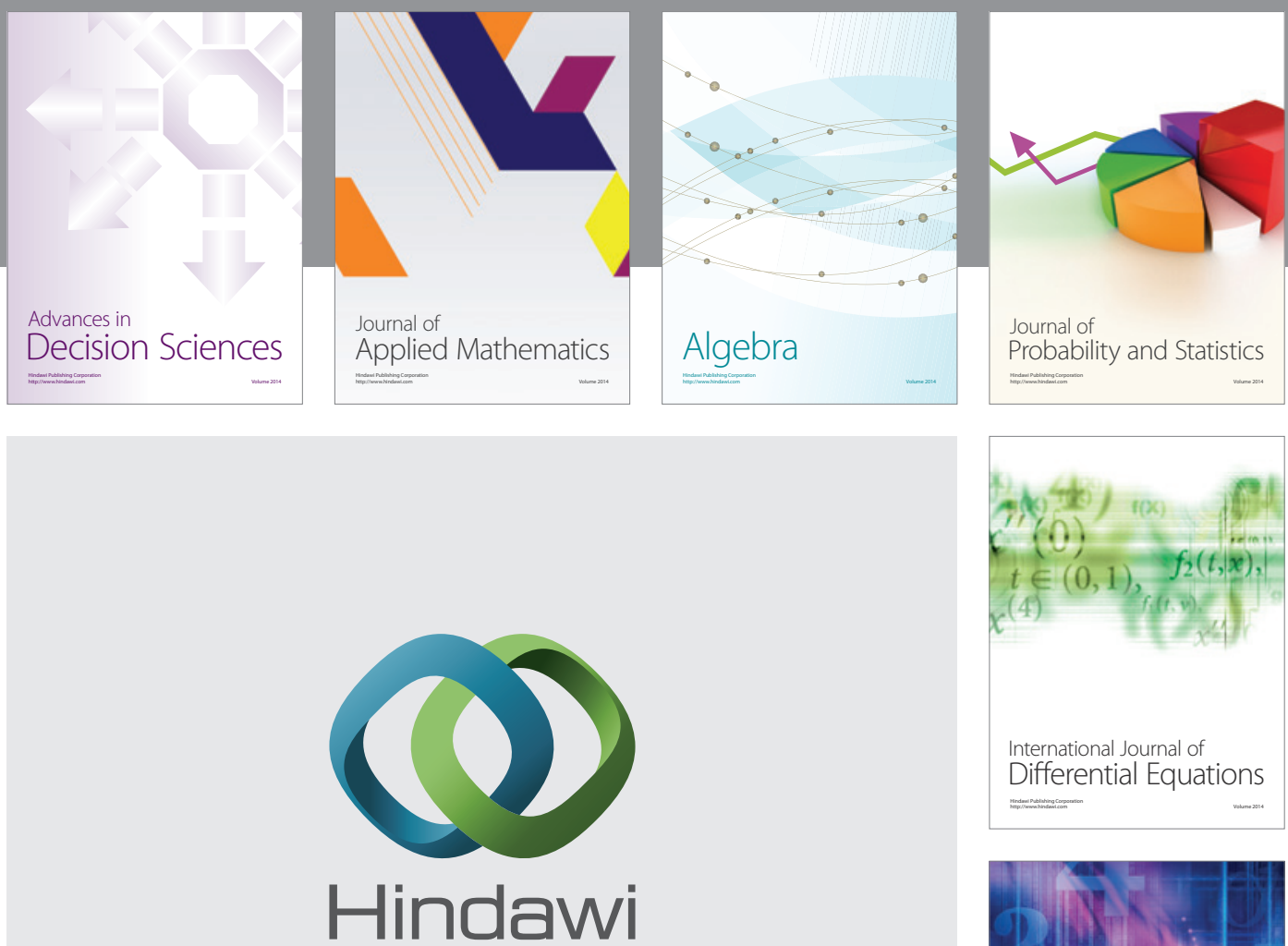

Submit your manuscripts at http://www.hindawi.com
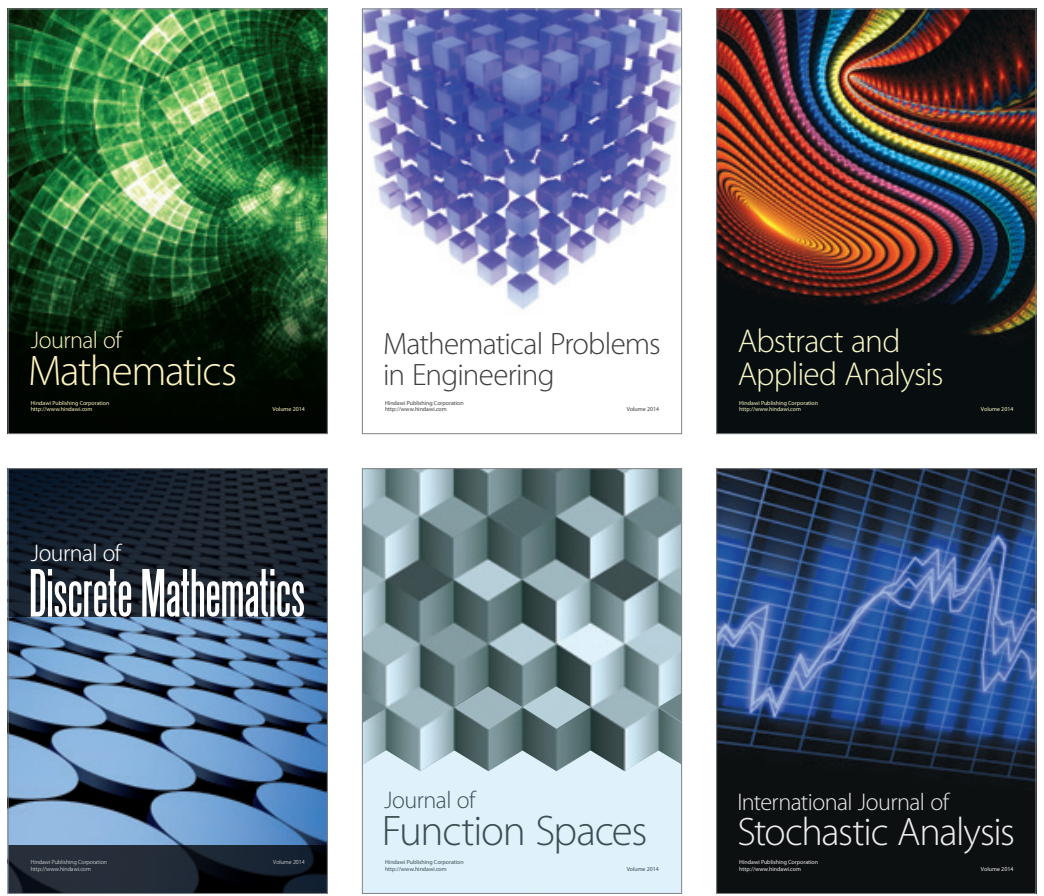

Journal of

Function Spaces

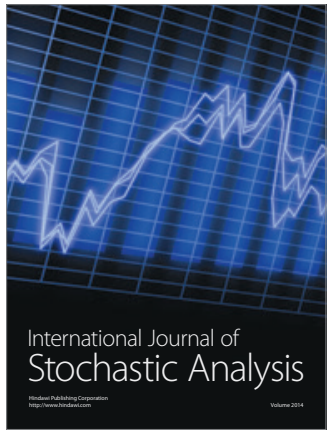

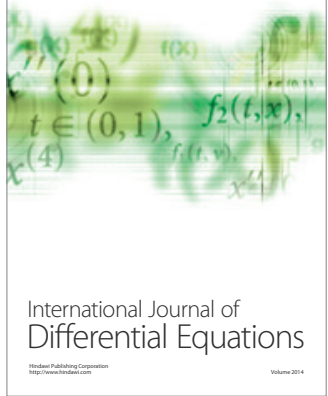
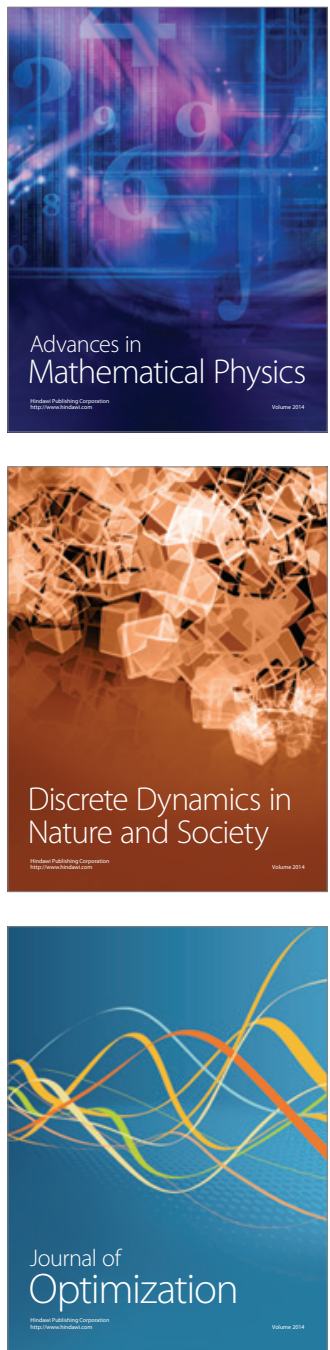